\title{
APRENDIZAJE Y COMPETENCIAS. UNA NUEVA MIRADA
}

\author{
LEARNING AND COMPETENCES. A NEW VIEW \\ Fuensanta Hernández Pina*, Pilar Martínez Clares**, \\ Mirian Martínez Juárez***, Fuensanta Monroy Hernández**** \\ Universidad de Murcia
}

\begin{abstract}
RESUMEN
Los retos más importantes a los que se enfrenta la sociedad del Siglo XXI es el aprendizaje a lo largo de la vida y la formación basada en competencias. El aprendizaje ya no se ve como un proceso pasivo que se lleva a cabo en espacios formales, sino como algo que tiene lugar a lo largo y ancho de la vida. Ahora más que nunca es necesario un aprendizaje continuo y permanente y este cambio demanda un modelo de educación centrado en el aprendizaje y en la formación integral y basada en competencias. Entorno a toda esta corriente se desarrolla un interesante corpus de investigaciones, teorías y modelos que sitúan al aprendiz (estudiante) como "el actor social protagonista" de este proceso. Respecto a las competencias, término actualmente tan complejo como poliédrico, se aboga por una formación integral basada en las mismas con la intención, de capacitar a las personas no sólo para el presente sino para los futuros retos con los que se deberá enfrentar. En este trabajo se presenta una nueva visión de estos dos elementos, tan necesarios y emergentes en el Proceso de Bolonia, en el que estamos inmersos. Para ello hemos hecho una integración de las distintas dimensiones de la Competencia de Acción Profesional (C.A.P.) en las concepciones cualitativas y cuantitativas del aprendizaje y la enseñanza.
\end{abstract}

Palabras clave: Enseñanza, Aprendizaje, Concepciones de Enseñanza y Aprendizaje, Competencias.

\section{ABSTRACT}

The most important challenges faced by today's society is lifelong learning and competence-based training. Learning is no longer seen as a passive process carried out in formal environments, but should be reconceptualised as a lifelong and life-wide development. Continuous, permanent training is

\footnotetext{
* Es Catedrática de Método de Investigación y Diagnostico en Educación, E-mail: fhpina@um.es.

** Profesora Titular de Método de Investigación y Diagnostico en Educación.

*** Profesora contratada de Método de Investigación y Diagnostico en Educación.

**** Es Máster en Psicopedagogía y estudiante de doctorado del Departamento de Método de Investigación y Diagnostico en Educación.
} 
more important than ever before and these changes demand an educational model based on learning and on comprehensive, competence-based training. In order to explain such a phenomenon, a number of research studies, theories and models have been carried out, which position the learner as "main social actor" of this process. With regard to competences (term coined nowadays which seems to be both complex and multifunctional), comprehensive training based on competences is fostered, which enables people to confront, not only current events but also future challenges. This paper presents a new vision of these two aspects bearing in mind the importance of learning and competences in the Bologna Process. In order to do so, we integrated the different dimensions of the Professional Action Competence (C.A.P. for short) and qualitative and quantitative conceptions of learning and conceptions of teaching.

Key words: Teaching, Learning, Conception of Teaching and Learning, Competences.

\section{Introducción}

Los retos más importantes con los que se enfrenta la sociedad actual y futura son la construcción del aprendizaje y la formación en competencias de acción. En el nuevo discurso político de la Comisión Europea, la construcción del aprendizaje y el desarrollo de las competencias, se perciben como una de las mejores inversiones para el desarrollo del capital humano, alzando la mirada más allá de los espacios formales y de las competencias académicas propiamente dichas. En esta línea nos encontramos con el informe Delors (1996) en el que ya se señala que la sociedad del siglo XXI será una sociedad cognitiva, en la que el capital más importante será el capital de conocimientos avanzados y de las competencias para resolver problemas y crear soluciones nuevas.

A este informe le han seguido los retos que plantea el Proceso de Bolonia. El aprendizaje y la adquisición de competencias y habilidades están entre los desafíos más importantes de dicho Proceso de Bolinia. Su implantación está suponiendo trasladar el centro de atención del profesor al estudiante y de pasar de una formación basada en los conocimientos exclusivamente a otra basada en la adquisición de competencias académicas, profesionales y de acción. Este nuevo marco está teniendo consecuencias para la nueva estructuración (el sistema de cualificaciones) de la universidad pero, sobre todo, para el docente que tiene que cambiar su concepción de la enseñanza integrando el aprendizaje en el binomio enseñanzaaprendizaje, convirtiéndolo en un proceso más bidireccional. Tanto el profesor como el estudiante deberán pasar de una concepción puramente cuantitativa a otra cualitativa de nivel alto. El EEES supone un replanteamiento docente diferente puesto que otorga a los estudiantes un protagonismo diferente en el proceso de enseñanza-aprendizaje. Exige además adoptar concepciones diferentes por parte de ambos, estudiantes y profesores, ya que como destaca Caballero Hernández-Pizarro (2007) el profesor no podrá limitar su preocupación por los contenidos científicos, sino que ha de reflexionar sobre el modo en que los conocimientos son aprendidos y el modo en que pueden ser enseñados.

En el artículo de Niemi (2009), síntesis de la conferencia plenaria impartida en ECER 2008, se señala que para conocer los nuevos planteamientos sobre el aprendizaje debemos proceder a realizar investigación de mayor calado y calidad y desde planteamientos multidisciplinares. La investigación actual sobre aprendizaje está demostrando la capacidad de empoderamiento que éste tiene para el desarrollo de las personas y cómo el aprendizaje se está produciendo en contextos tan diferentes que están rompiendo las fronteras de lo que tradicionalmente se han considerado los espacios del aprendizaje. La investigación sobre 
aprendizaje y la educación deben estar en una simbiosis permanente. De hecho son varios los programas que sobre aprendizaje y enseñanza se está desarrollando en varios países de Europa, como es el Programa CICERO (Cross-disciplinary Initiative for Collaborative Efforts of Research on Learning) en Finlandia; el programa TLRP (Teaching and Learning Research Programme) en UK; el programa PROO (Programma Oderwijsonderzoek-Educational Research Program) en los Países Bajos y el programa 'Knowledge, Education and Learning' en Noruega.

Todos los cambios que se están produciendo exigen una nueva formación en conocimientos, habilidades, competencia y valores porque la sociedad demanda nuevas competencias y nuevas formas de aprender. La gran transformación estructural de nuestros días origina cambios en la economía, la tecnología y la sociedad y producen un nuevo escenario y, por tanto, nuevos planteamientos en la formación y en el aprendizaje. El nuevo concepto de aprendizaje se centra en los resultados del proceso en términos de competencias adquiridas.

\section{Desarrollo}

\section{a) Aprendizaje}

Tanto el concepto de enseñanza como el de aprendizaje han evolucionado a lo largo de las últimas décadas en varias de sus dimensiones, desde una visión positivista, individual, pasiva y formal a otra más colectiva, activa, autorregulada y contextual. Sfard (1998) identifica dos metáforas para referirse a ambas posiciones sobre la conceptualización del aprendizaje, la metáfora de la adquisición y la metáfora de la participación. Cada una de ellas supone modos diferentes de pensar, actuar y actividades diferentes y, por consiguiente, en una fase de transferencia posterior, a modos diferentes de actuar en la vida cotidiana.

En las últimas décadas los estudios sobre la enseñanza y el aprendizaje experimentan un nuevo e interesante impulso, que propician un corpus de conocimientos y resultados de investigación que dan lugar a nuevos modelos para explicar y estudiar estos dos factores. Estos modelos han sido los enfoques de aprendizaje o Student Approaches to Learning (SAL) (Marton y Säljö, 1976 a, b; Biggs, 1987; Kember, 2000; Trigwell y Prosser, 1991; Hernández Pina, 1993, 1998, 2002, 2004, 2005) y el aprendizaje autorregulado (Self-Regulated Learning) (Pintrich, 2004).

El modelo de enfoques de aprendizaje (SAL), que empieza a configurarse en los años 1970, tiene su base en el constructivismo donde el centro de interés se proyecta en la comprensión que realizan los estudiantes cuando aprenden, frente a la memorización.

Los mayores beneficiados de esta nueva corriente de estudio son el profesorado ya que, tanto el modelo de aprendizaje como el modelo de enseñanza desarrollados por Biggs (1987), Prosser, Ramsden, Trigwell y Martín (2003), permiten conocer los factores que más afectan, por un lado, a los enfoques de enseñanza y aprendizaje y, por otro, a la calidad de los resultados del aprendizaje, y sirven para mejorar la práctica y las estrategias pedagógicas (Hernández Pina, 2005).

Las concepciones sobre la enseñanza y el aprendizaje, por otro lado, han sido objeto de mucha investigación cualitativa a lo largo de las tres últimas décadas (Kember, 1997; Hernández Pina, 2009). En esta línea de trabajo, el conocimiento y la realidad son percibidas de 
forma diferente, tanto por profesores como por estudiantes, a lo largo y ancho de los niveles educativos, contextos y países diferentes. La cultura, el contexto social, la propia historia, la experiencia propia, etc. son factores muy determinantes y están en la base para interpretar la información, la realidad y crear nuevos significados. Diferentes lentes permiten ver la misma realidad de forma diferente. La conclusión generalizada es que las concepciones son dinámicas y dependen de las epistemologías que cada sujeto sostenga y del contexto sociocultural.

El modo en que los profesores y los estudiantes enfocan su enseñanza y el aprendizaje va a depender de la concepción que ambos tengan de estos dos factores (Trigwell y Prosser, 1996). Aquellos que conceptualizan la enseñanza como una transmisión de la información percibirán al profesor como el protagonista principal del proceso. En cambio, los que la conceptualicen como una construcción del conocimiento situarán al estudiante en el centro del proceso.

Hasta finales del XX se considera que el espacio natural del aprendizaje es la educación formal. Hoy los espacios de aprendizaje van más allá y adquieren gran relieve los llamados espacios no formales, informales que se convierten en espacios de auténtico aprendizaje. El aprendizaje permanente propicia la valoración de otros espacios de aprendizaje fuera de las instituciones educativas, fuera de los escenarios puramente escolares o académicos, de tal forma que se vuelen complementarios a los espacios formales. Los contextos del aprendizaje informal y no formal son claves en el desarrollo del concepto de aprendizaje a lo largo de la vida. Niemi (2009) propone utilizar el término life-wide learning para referirse no a lo largo de la vida sino añadir a lo ancho de la misma.

Una de las conclusiones del proyecto CERI (CERI, 2008; Niemi, 2003, 2009) revela que el aprendizaje es una de las mejores herramientas para la mejora de la calidad de vida. Los resultados sociales del aprendizaje tienen implicaciones que superan a las medidas económicas. El aprendizaje puede producir efectos que van más allá de las medidas que se utilizan como resultados de las inversiones económicas en la educación, especialmente en muchos aspectos de la vida cotidiana que aún no han sido sometidos a procesos de valoración. De acuerdo con autores como Niemi (2009) el aprendizaje es el mejor medio de empoderamiento y engrandecimiento de las personas para poder enfrentarse a las transformaciones presentes y futuras, puesto que a través del aprendizaje se pueden crear nuevos conocimientos y competencias.

\section{b) Las competencias}

La sociedad actual se ve envuelta en una serie de profundas transformaciones y cambios a consecuencia de nuestra integración en el Espacio Europeo que abarcan no sólo lo económico sino también lo educativo, lo cultural, lo formativo, etc. Todos estos cambios que se producen en nuestro entorno como es la globalización, la transnacionalidad de los mercados, la importancia de la formación integral, la multiculturalización de la sociedad, etc. reclaman un nuevo escenario que hace necesario y prioritario generar o replantear formas diferentes en la forma de afrontar la realidad, puesto que el saber, el saber hacer y el saber actuar son capacidades que deben desarrollarse y mantenerse actualizadas más allá de lo que hemos entendido hasta ahora como formación inicial (Martínez Clares, 2009). 
Ante estos aires de cambio, la sociedad se enfrenta a nuevos retos y desafíos en el conocido EEES. Los objetivos a los que nos enfrentamos son los de lograr que los estudiantes adquieran una formación integral y que el aprendizaje sea conceptualizado como algo que tiene lugar a lo largo de la vida. Esto supone crear un paradigma diferente en un entorno nuevo, en el que el desarrollo de capacidades, habilidades, actitudes y valores (la formación en competencias) tenga lugar en un nuevo enfoque de gestión y desarrollo del aprendizaje permanente, en el que se haga posible aprender a aprender, adaptarse a los cambios sociales, productivos, económicos y tecnológicos, y a transformar la realidad en la que se está inmerso. Por añadidura esto supone además que el egresado/profesional sea capaz de aprender de forma continua y autónoma a lo largo de la vida.

Hemos pasado de una enseñanza basada en una sociedad industrial a otra basada en el conocimiento y la información en la que se promueve el aprender a aprender, aprender a lo largo y ancho de la vida, el desarrollo de competencias, el aprender a convivir en una sociedad diversa y la construcción moral y ética.

Este escenario demanda una educación basada en competencias; es decir, una educación que haga posible el desarrollo de competencias profesionales y una educación que garantice la comprensión de lo que se transmite (Hernández Pina y cols., 2005).

Las competencias son un campo ampliamente abordado y pasa a ser un concepto omnipresente en todos los espacios sociales y educativos. Todos los cambios y transformaciones señalados, exigen un cambio en los modelos de formación centrados en el trabajo y el aprendizaje a través del desarrollo de competencias, que hagan posible el aprendizaje continuo a lo largo y ancho de la vida y la adaptación al contexto y la pluralidad que hoy presenta la sociedad.

La adquisición de las competencias hay que plantearlas desde un enfoque holístico que las concibe como el conjunto de conocimientos, procedimientos, capacidades y actitudes, complementarios entre sí de tal forma que los profesionales han de saber, saber hacer, saber estar y saber ser para actuar con la máxima eficacia y eficiencia en sus actuaciones profesionales. Las competencias, además, deben ser definidas en la acción o lo que es lo mismo en las acciones del trabajo. Las competencias, igualmente, deben ser situadas en el contexto para que puedan responder a situaciones dadas y a niveles requeridos en el trabajo. Por último, en la adquisición de las competencias la experiencia juega un papel fundamental para el desarrollo de las mismas (Echeverría, 2005 y Martínez Clares y Echeverría, 2009).

Todas estas características hacen alusión a las Competencias de Acción Profesional (C.A.P.) que se determinan (Martínez Clares y Echeverría, 2009) por: el saber actuar - no sólo por el cúmulo de conocimientos-, por estar contextualizada — exigencias, restricciones y recursos del entorno concreto donde se desarrolla-, por ser un saber actuar validado - demostrado en la acción y comprobado por otros - y por poseer una finalidad — con un sentido para la persona- (Le Boterf, 2001).

Una formación basada en competencias debe garantizar el saber, que lleve al profesional al dominio integrado de los conocimientos teóricos y prácticos de un puesto de trabajo (desarrollo profesional); el saber hacer, donde los procedimientos que se poseen garanticen la calidad productiva cuando se entremezclan con los saberes. Y el saber ser y estar o dominio de la cultura del trabajo y del ámbito social que se une a la participación en los asuntos productivos y sociales. 
El nuevo Espacio Europeo plantea un nuevo enfoque que aporta importantes transformaciones e innovaciones en pro de una formación de calidad, que permite la creación de un contexto de formación continua, que facilita la cooperación, la interacción dinámica y activa de todos los agentes implicados y la adquisición de competencias profesionales y de acción, que potencian la cualificación y la profesionalidad (CCU, 2000).

A través de este nuevo enfoque de gestión de la formación profesional continuo (Figura 1) se potencia la figura de los centros de formación como facilitadores, mediadores y orientadores de dicha formación para que potencien la gestión y la construcción del saber, del saber hacer y del saber ser y estar mediante la formación en competencias generales o transversales y específicas propias de cada puesto de trabajo o grupos de actuaciones profesionales. En definitiva, este enfoque apuesta por formar personas que puedan pensar por sí mismas y aprender por sí mismas.

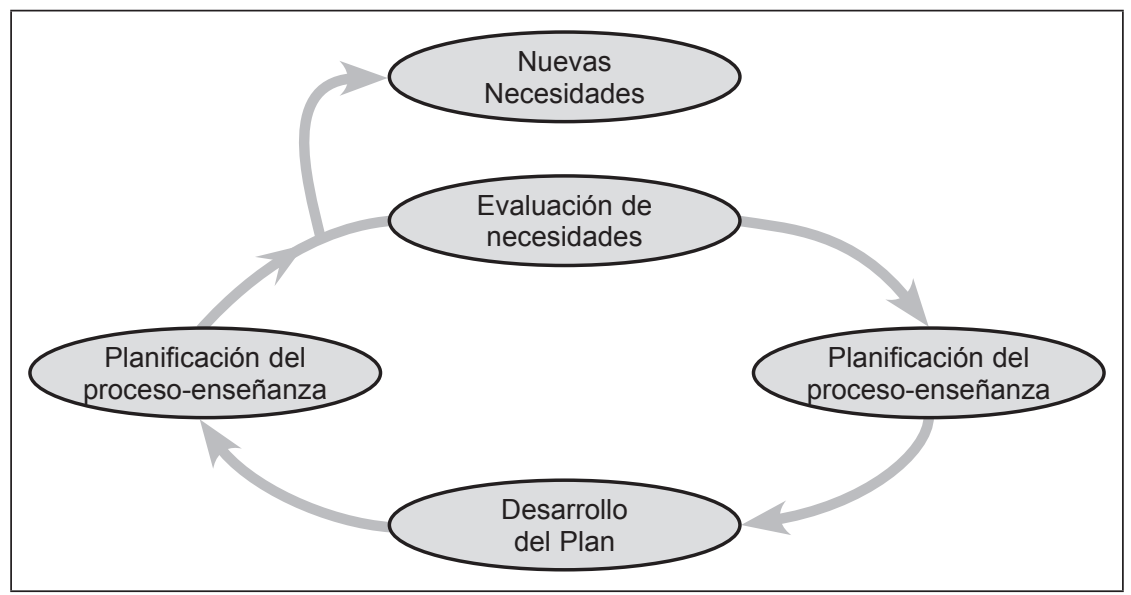

FIGURA 1.

Desarrollo profesional continuo.

Para ello es importante definir las competencias vinculadas con cada perfil profesional, constituyendo éstas el conjunto de conocimientos, competencias y funciones que se desarrollan al final de un proceso formativo, y que permitirán a la persona manejarse con éxito en diferentes situaciones de su quehacer laboral a lo largo de su proyecto profesional (Martínez Clares y cols, 2008).

Para terminar queremos hacer las propuestas de integración de las cuatro dimensiones de la Competencia de Acción Profesional, analizadas someramente a lo largo de este trabajo. El desarrollo de estas cuatro dimensiones, será posible en una concepción del aprendizaje y de la enseñanza de corte cualitativo; es decir, el desarrollo de las competencias será viable en un programa formativo con concepciones de aprendizaje y de enseñanza que vayan más allá de la mera transmisión del conocimiento y la información. Esta integración la podemos observar esquemáticamente en la Tabla 1, donde en una concepción de corte cuantitativo sólo cabe la competencia del saber. Conforme avanzamos hacia concepciones más cualitativas se van haciendo presentes las otras tres competencias de forma simultánea. 
TABLA 1. Relación entre competencias, concepciones, enseñanza y aprendizaje.

\begin{tabular}{|l|l|l|l|}
\hline Competencias & Concepciones & \multicolumn{1}{|c|}{ Enseñanza } & \multicolumn{1}{c|}{ Aprendizaje } \\
\hline Saber & $\begin{array}{l}\text { Impartición de la } \\
\text { información } \\
\text { Transmismitión el } \\
\text { conocimiento } \\
\text { estructurado }\end{array}$ & $\begin{array}{l}\text { Adquisición de conocimientos } \\
\text { Adquisición de conocimientos a tra- } \\
\text { vés de procedimientos que permitan } \\
\text { la comprensión. }\end{array}$ \\
\hline Saber hacer & $\begin{array}{l}\text { Comprensión/ } \\
\text { competencias }\end{array}$ & $\begin{array}{l}\text { Interacción profesor- } \\
\text { alumno } \\
\text { Facilitador de la } \\
\text { comprensión }\end{array}$ & $\begin{array}{l}\text { El aprendizaje es adquisición de he- } \\
\text { chos y procedimientos para utilizar } \\
\text { cuando se necesitan }\end{array}$ \\
\hline Ser & Cambio & $\begin{array}{l}\text { Desarrollo intelectual } \\
\text { y cambio conceptual }\end{array}$ & $\begin{array}{l}\text { El aprendizaje viene facilitado por el } \\
\text { profesor }\end{array}$ \\
\hline Estar & Cambio & $\begin{array}{l}\text { El aprendizaje significa exponerse a } \\
\text { las actividades que generan la com- } \\
\text { prensión de los contenidos de la ma- } \\
\text { teria y el desarrollo de competencias } \\
\text { El aprendizaje sirve para cambiar y } \\
\text { transformar a la persona }\end{array}$ \\
\hline
\end{tabular}

La universidad del siglo XXI y del EEES exige un nuevo contexto en el que sea posible una concepción de la enseñanza y del aprendizaje que se enriquezca y engrandezca desde el enfoque co-constructivista. Un modelo en el que el contexto adopte un nuevo valor y así el estudiante adquiera la capacidad de percibir los problemas desde perspectivas diferentes, que aprenda a analizar, sintetizar, a ser más flexible, etc. En síntesis, que aprenda las competencias que le ayuden desarrollarse intelectualmente y lograr cambios conceptuales transformarse como persona. Es decir, que sean capaces, no de poder con el mundo que les rodea sino de prosperar y saber vivir en él.

\section{Referencias Bibliográficas}

Biggs, J. (1987). Student approaches to learning and studying. Melburne: Australian Council for Educational Research.

Caballero, M. A (2007). "La adaptación al Espacio Europeo de Educación Superior como escenario para la reflexión sobre el proceso de enseñanza-aprendizaje en el ámbito universitario: algunas propuestas para un cambio significativo". REOP, Vol. 18 (2), 167-177.

CCU. (2000). Memorándum sobre el aprendizaje permanente. COMISIÓN DE LAS COMUNIDADES EUROPEAS. Bruselas, 30.10.2000, SEC (2000) 1832.

Centre for Educational Research and Innovation (CERI) (2008) More about the Social Outcomesn of Learning Projet. http://www.oecd.org/document/35/0,3343,en_2649_35845581_35762147_1_1_1 _ 1,00.html.

Delors, J. (Coord.) (1996). La educación encierra un tesoro. Madrid: Santillana. UNESCO.

Echeverría, B. (2005). Competencia de acción de los profesionales de la orientación. Madrid: ESIC. 
Hernández Pina, F. (2004). Enseñar y aprender en la Universidad: ¿Qué enseñar? ¿Qué aprender? Actas del III Symposium Iberoamericano de Docencia Universitaria Pedagogía Universitaria: Hacia un Espacio de Aprendizaje Compartido. ICE de Universidad de Deusto, pp. 81-93.

Hernández Pina, F. (2009). University academics 'and students' conceptions of teaching and learning in Higher Education. Comunicación presentada ECER, Viena.

Hernández Pina, F. y Martínez Clares, P. (2008). La formación en competencias en educación superior: un nuevo factor de calidad en el contexto multicultural. En E. Soriano (Coord.). Educar para la ciudadanía intercultural y democrática. Madrid: La Muralla.

Hernández Pina, F.; Martínez Clares, P.; Da Fonseca Rosario, P. y Rubio Espín, M. (2005). Aprendizaje, competencias y rendimiento en educación Superior. Madrid: La Muralla.

Kember, D. (1997). A reconceptualisation of the research into university academics. conceptions of teaching. Learning and Instruction, 7(3), 255-275.

Le Boterf, G. (2001). Ingeniería de las competencias. Barcelona: Gestión 200, S.A.

Martínez Clares, P (2009). Formación basada en competencias. ¿Ser o tener competencias? Mesa Redonda: XIV Congreso Nacional de Modelos de Investigación Educativa. Educación. Huelva.

Martínez Clares, P. y Echeverría, B. (2009). "Formación basada en competencias". Revista de Investigación Educativa, Vol. 27, 1, 125-147.

Martínez Clares P.; Martínez Juárez, M. y Muñoz, M. (2008). “Aprendizaje por competencias en educación superior". Revista Galego-Portuguesa de Psicoloxia e educación. Vol, 16 (1-2), pp 193-213.

Niemi, H. (2003) Competence Building in Life-Wide Learning, in P. Conçeicăo, M. V. Heitor \& BÅ. Lundwall (Eds) Innovation and Competence Building with Social Cohesion for Europe, 219-239. Cheltenham: Edward Elgar.

Niemi, H. (2009). "Why from Teaching to Learning?" European Educational Research Journal. Vol. 8 (1). http://www.wwwords.co.uk/eerj/.

Prosser, M., Ramsden, P., Trigwell, K. y E. Martin. (2003). "Dissonance in experience of teaching and its relation to the quality of student learning". Studies is Higher Education, 28, 37-48.

Sfard, A. (1998). "On Two Metaphors for Learning and the Dangers of Choosing Just One". Educational Researcher, 27(2), 4-13.

Samuelowicz, K. y Bain, J. D. (1992). "Conception of teaching held by academia teachers". Higher Education, 24, 93-112.

Trigwell, K. y Prosser, M. (1996). "Changing approaches to teaching: a relational perspective". Studies is Higher Education, 21, 275-284.

UNESCO, (1998). "La educación superior en el siglo XXI: Visión y Acción". Conferencia Mundial sobre Educación Superior. París, Octubre 1998.

Fecha de recepción: 31-07-2009

Fecha de revisión: 13-10-2009

Fecha de aceptación: 21-10-2009 